\title{
A Practical Comparison of Phase Diversity to Interferometry in Measuring the Aberrations in an Adaptive Optics System
}

\author{
Don Gavel \\ Brian Bauman \\ Gene Campbell \\ Carmen Carrano \\ Scot Olivier
}

This paper was prepared for submittal to the

44th Annual Meeting of the International Symposium on Optical Science,

Engeering, and Instrumentation

Denver, $\mathrm{CO}$

July 18-23, 1999

July 1999

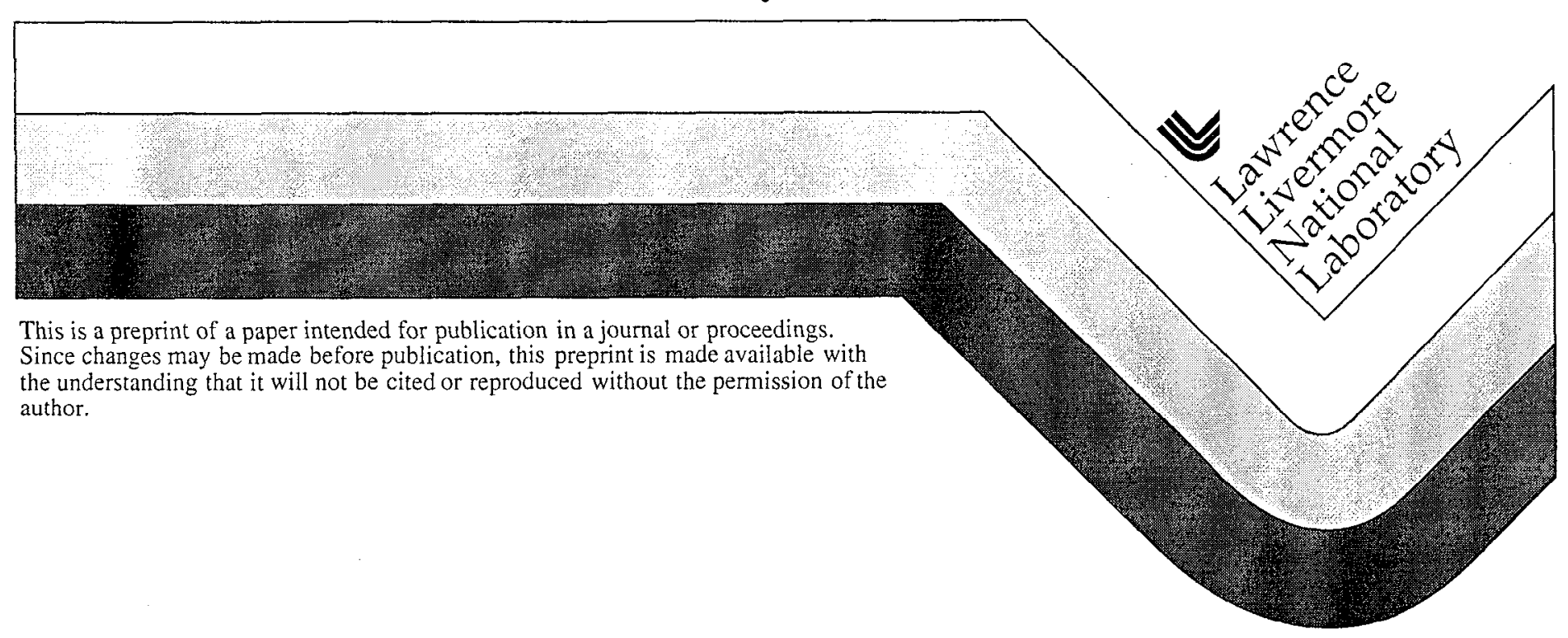




\section{DISCLAIMER}

This document was prepared as an account of work sponsored by an agency of the United States Government. Neither the United States Government nor the University of California nor any of their employees, makes any warranty, express or implied, or assumes any legal liability or responsibility for the accuracy, completeness, or usefulness of any information, apparatus, product, or process

disclosed, or represents that its use would not infringe privately owned rights. Reference herein to any specific commercial product, process, or service by trade name, trademark, manufacturer, or otherwise, does not necessarily constitute or imply its endorsement, recommendation, or favoring by the United States Government or the University of California. The views and opinions of authors expressed herein do not necessarily state or reflect those of the United States Government or the University of California, and shall not be used for advertising or product endorsement purposes. 


\title{
A Practical Comparison of Phase Diversity to Interferometry in Measuring the Aberrations in an Adaptive Optics System
}

\author{
Don Gavel, Brian Bauman, Gene Campbell, Carmen Carrano, and Scot Olivier \\ Lawrence Livermore National Laboratory \\ 7000 East Avenue, MS L-395, Livermore, CA 94550
}

\begin{abstract}
Any adaptive optics system must be calibrated with respect to internal aberrations in order for it to properly correct the starlight before it enters the science camera. Typical internal calibration consists of using a point source stimulus at the input to the $\mathrm{AO}$ system and recording the wavefront at the output. Two methods for such calibration have been implemented on the adaptive optics system at Lick Observatory. The first technique, Phase Diversity, consists of taking out of focus images with the science camera and using an iterative algorithm to estimate the system wavefront. A second technique uses a newly installed instrument, the Phase-Shifting Diffraction Interferometer, which has the promise of providing very high accuracy wavefront measurements. During observing campaigns in 1998, both of these methods were used for initial calibrations. In this paper we present results and compare the two methods in regard to accuracy and their practical aspects.
\end{abstract}

Keywords: $\quad$ Adaptive Optics, Calibration, Phase Diversity, Diffraction Interferometry

\section{INTRODUCTION}

Optical alignment alone is not sufficient for an AO system to provide diffraction-limited images to the science camera at its focus. The internal aberrations in the system must be calibrated out to high accuracy beforchand. The Lick AO system can presently use onc of two techniques to calibrate: 1) phase diversity using out-of-focus images taken with the science camera, and 2) phase-shifting point diffraction interferometetry.

\section{PHASE DIVERSITY}

Phase diversity has been used for a number of years to establish internal calibration of the adaptive optics system at Lick ${ }^{1}$. The technique involves taking an out-of-focus image at the science focus and using this data in an iterative computer algorithm. The algorithm determines a pair of complex wavefronts at both the pupil and the (out-of-focus) image planes consistent with the assumptions that the pupil is uniformly illuminated and that the intensity at the (out-of-focus) image plane is the measured intensity. The usual approach in the calibration procedure is then to apply the phase diversity solution (phase at the pupil) to the deformable mirror. The process of measurement and correction is then repeated with the objective of achieving the highest possible Strehl Airy pattern in the (in-focus) image. 
The technique has been used successfully at Lick to adjust actuators on the deformable mirror to within $100 \mathrm{~nm}$ rms of flat.

\section{POINT DIFFRACTION INTERFEROMETRY}

The interferometer has just recently been installed on the Lick AO system. It uses Phase-shifting Point Diffraction Interferometry (PSDI) technology developed originally for the Extreme Ultraviolet Lithography Program at LLNL. The technique is described in detail in a companion paper ${ }^{2}$. Essentially, the approach is to build a Twyman-Green interferometer using fiber optics. A stable, long coherence length laser is split into two fibers: one fiber feeds the input of the AO system at the telescope focus; the other fiber feeds in at the AO system output focus near the science camcra. The wave from the AO system converges at and reflects off of the polished end of the second fiber, interfering with the spherical wave emitted by it. A scraper mirror sends an image of the resulting interference pattern to a digital camera and frame-grabber.Phase-shifting is accomplished by stretching the fiber in one arm with a piezo-electric transducer. The system uses a 12-bin algorithm to recover absolute phase in a manner similar to conventional phase shifting interferometers.

\section{A COMPARISON}

Figure 1 compares phase diversity to PSDI measurements of internal AO system phase, taken at the beginning of a night's observing at Lick. The source is a HeNe laser $(632.5 \mathrm{~nm})$ fed from a fiber source small enough to appear diffraction-limited at the input focus of the adaptive optics system. The map of wavefront phase perturbations shows the characteristic "orange peel" surface of the deformable mirror, with the hexagonal geometry of actuator placements being readily apparent. Except for a few drop-out pixels (possibly due to loss of illumination) near the edge of the PSDI image, the two measurements very closely agree. PSDI has more spatial resolution (it uses a 1024x1024 array, as opposed to $256 \times 256$ in the phase diversity measurement) and hence has sharper features. The measurement shows one actuator in the upper left is too low, and one in the lower left is too high. Subsequent application of these corrections to the deformable mirror corrected the wavefront to better than $100 \mathrm{~nm} \mathrm{rms}$. 
a

Figure 1.

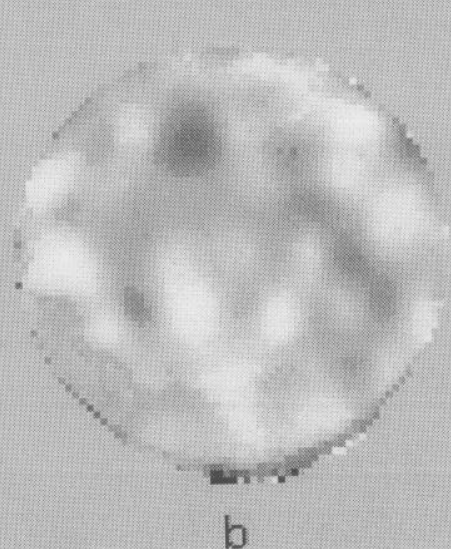

b

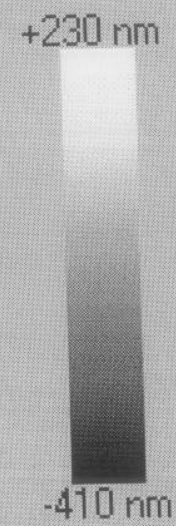

\section{Residual Phase in the Lick Adaptive Optics System as measured by (a) phase diversity and (b) phase shifting diffraction interferometry}

\section{CONCLUSION}

Both phase diversity and phase-shifting diffraction interferometry are practical techniques for achieving accurate internal calibration of the Lick adaptive optics system. Both are capable of measuring to well beyond the accuracy to which the deformable mirror can be flattened, about 90 nanometers rms over the pupil. PSDI is relatively new to the system and offers a number of advantages. For example, PSDI quickly gives a direct readout of phase, whereas the phase diversity approach requires waiting for computer iterations to converge. An important advantage of PSDI is that it is not susceptible to two-pi phase ambiguity, while phase diversity breaks down if the phase varies by more than a wave over the aperture. This issue is quite important particularly at the initial stages of calibration (say, at the beginning of a set of observing nights just after having mounted the system on the telescope), when the deformable mirror usually starts out worse than a wave out of flat. The only way to get phase diversity started is physically remove the deformable mirror and put in its place a flat mirror, record the resulting Hartmann sensor readings, put the deformable mirror back in place, close the loop and record the resulting "flattening" commands to the mirror. Removing and replacing the deformable mirror is both time consuming and risky, although usually only necessary once in a run.

\section{ACKNOWLEDGMENT}

This work was performed under the auspices of the U. S. Department of Energy by the Lawrence Livermore National Laboratory under contract number W-7405-ENG-48. It was supported by Laboratory Directed Research and Development funding within the University Relations Program and the Lasers Directorate. This support is gratefully acknowledged 


\section{REFERENCES}

1. Carrano, C. J., Olivier, S. S., Brase, J. M., Macintosh, B. A., An, J. R., Phase retrieval techniques for adaptive optics, Proc. SPIE Vol. 3353, September, 1998, 658-667.

2. E. W. Campbell, B. J. Bauman, D. R. Sweider, S. S. Olivier, High-accuracy calibration of an adaptive optics system using a phase shifting diffraction interferometer, Proc. SPIE 3762, July 1999 [this conference]. 